\title{
Work Statistics across a Quantum Phase Transition
}

\author{
Zhaoyu Fei, ${ }^{1}$ Nahuel Freitas $\odot,{ }^{2}$ Vasco Cavina, ${ }^{2}$ H. T. Quan, ${ }^{1,3,4,}$ and Massimiliano Esposito ${ }^{2, \dagger}$ \\ ${ }^{1}$ School of Physics, Peking University, Beijing 100871, China \\ ${ }^{2}$ Complex Systems and Statistical Mechanics, Physics and Materials Science, University of Luxembourg, \\ L-1511 Luxembourg, Luxembourg \\ ${ }^{3}$ Collaborative Innovation Center of Quantum Matter, Beijing 100871, China \\ ${ }^{4}$ Frontiers Science Center for Nano-optoelectronics, Peking University, Beijing, 100871, China
}

(Received 18 February 2020; accepted 15 April 2020; published 1 May 2020)

\begin{abstract}
We investigate the statistics of the work performed during a quench across a quantum phase transition using the adiabatic perturbation theory when the system is characterized by independent quasiparticles and the "single-excitation" approximation is assumed. It is shown that all the cumulants of work exhibit universal scaling behavior analogous to the Kibble-Zurek scaling for the average density of defects. Two kinds of transformations are considered: quenches between two gapped phases in which a critical point is traversed, and quenches that end near the critical point. In contrast to the scaling behavior of the density of defects, the scaling behavior of the cumulants of work are shown to be qualitatively different for these two kinds of quenches. However, in both cases the corresponding exponents are fully determined by the dimension of the system and the critical exponents of the transition, as in the traditional Kibble-Zurek mechanism (KZM). Thus, our study deepens our understanding about the nonequilibrium dynamics of a quantum phase transition by revealing the imprint of the KZM on the work statistics.
\end{abstract}

DOI: 10.1103/PhysRevLett.124.170603

Introduction.-In cosmology and condensed matter physics the creation of excitations during continuous phase transitions (thermal or quantum) is usually described by the Kibble-Zurek mechanism (KZM) [1-4]. The KZM relates the average density $\left\langle n_{\mathrm{ex}}\right\rangle$ of excitations or defects created during a transformation or quench across a critical point to the rate or speed at which the critical region is traversed. This is particularly relevant for adiabatic quantum computation and simulation schemes, where nonadiabatic effects impose a tradeoff between the speed and the fidelity that can be achieved [5-7]. Importantly, the KZM predicts a universal power law dependence of $\left\langle n_{\mathrm{ex}}\right\rangle$ on this rate, with an exponent that is fully determined by the dimension of the system and the critical exponents of the transition. Also, the KibbleZurek scaling of the irreversible entropy production has been reported [8]. The traditional heuristic argument behind the KZM, as well as more rigorous derivations based on the adiabatic perturbation theory [9-11], only gives information about the average density of excitations $\left\langle n_{\mathrm{ex}}\right\rangle$. However, the actual number of excitations created during a particular realization of the quench is a stochastic quantity that will fluctuate from one realization to the next, and thus must be characterized by a probability distribution. It was recently shown by del Campo that in the exactly solvable one dimensional (1D) transverse Ising chain the universal scaling predicted by the KZM also applies to all the cumulants of $n_{\mathrm{ex}}$ [12], which was recently verified in experiments [13].
Motivated by this finding, we extend previous results in two important aspects. In the first place, we turn our attention away from the density of created excitations and focus instead on the amount of work applied during the quench. Thus, we investigate what are the signatures of the KZM on the characteristic function of work (CFW), which plays an important role in the newly developed field of stochastic thermodynamics [14-16]. In analogy to the partition function, which contains essential information about an equilibrium state, the CFW contains essential information about an arbitrary nonequilibrium process. This interesting quantity has received much attention since it allows us to understand the emergence of irreversibility during a thermodynamic transformation (via the fluctuation relations $[17,18])$, and is related to other interesting quantities employed to study the nonequilibrium dynamics of complex many-body systems like the Loschmidt echo [18-22]. Second, we provide a general scaling argument, underpinned by the well-known results in the adiabatic perturbation theory [9-11], showing that all the cumulants of the work exhibit a scaling behavior similar to the KZM scaling for systems that can be described in terms of independent quasiparticles. Our predictions are valid in principle for systems in arbitrary dimensions, and are explicitly shown to hold in the exactly solvable 1D quantum transverse Ising model.

KZM and the adiabatic perturbation theory.-We first briefly review the basic concepts and heuristic arguments behind the KZM scaling in a quantum phase transition, and 
also how to recover (and generalize) the same results using the adiabatic perturbation theory. We consider a secondorder quantum phase transition between two gapped phases characterized by the correlation length critical exponent $\nu$ and the dynamic critical exponent $z$ [23]. Thus, close to the quantum critical point, the energy gap $\Delta$ between the ground state and the first relevant excited state, the relaxation time $\tau$ and the correlation length $\xi$ scale as [24]

$$
\Delta \sim|\lambda|^{z \nu}, \quad \tau \sim|\lambda|^{-z \nu}, \quad \xi \sim|\lambda|^{-\nu},
$$

where $\lambda$ is a dimensionless parameter which measures the distance from the critical point. We also consider a protocol in which the Hamiltonian of the system is modified in such a way that the parameter $\lambda(t)$ can be approximated as a linear quench $\lambda(t)=v t$ near the critical point, where $v>0$ is the quench rate. The system is initially prepared in the ground state at $t_{0} \rightarrow-\infty$ and the protocol stops at $t_{1} \rightarrow \infty$. According to the KZM, the evolution of the system can be divided into three parts: (1) $t_{0}<t<-t^{*}$, (2) $-t^{*}<t<t^{*}$, and (3) $t^{*}<t<t_{1}$, where the time $t^{*}$ is determined by the following argument $[3,4,24,25]$. During parts (1) and (3), the relaxation time of the system is sufficiently small for its evolution to be considered adiabatic $\left(\tau<v^{-1}\right)$, since it can always catch up with the change of $\lambda(t)$ (adiabatic region). In contrast, during part (2) the relaxation time is large compared to $v^{-1}$ and as a consequence the state of the system is frozen out (impulse region). The freeze-out time $t^{*}$ can be estimated by the following relation $t^{*} \simeq \tau\left[\lambda\left(t^{*}\right)\right]$ and thus we obtain $t^{*} \sim v^{-z \nu /(z \nu+1)}$. Then, the initial state for the adiabatic dynamics of part (3) is approximately the final state of the evolution of part (1), and is therefore characterized by a correlation length $\xi^{*}=\xi\left[\lambda\left(t^{*}\right)\right] \sim$ $v^{-\nu /(z \nu+1)}$. This correlation length corresponds to the characteristic length of the system, e.g., the size of the magnetic domains. Thus, the average density of defects or domain walls can be estimated as

$$
\left\langle n_{\mathrm{ex}}\right\rangle \sim \xi^{*-d} \sim v^{(d \nu) /(z \nu+1)},
$$

where $d$ is the dimension of the system.

The above results can be reproduced by using the adiabatic perturbation theory [9-11]. For this purpose, we consider a system defined on a $d$-dimensional lattice and described by a Hamiltonian $\hat{H}[\lambda(t)]=\hat{H}_{0}+\lambda(t) \hat{V}$, where $\hat{H}_{0}$ is the Hamiltonian at the quantum critical point and $\lambda(t)$, now called the work parameter [15], is controlled by an external agent according to the above protocol. Here, $\hat{V}$ is the driving Hamiltonian. We assume that the system can be described by independent quasiparticles (denoted by mode $k$ ), and that at the critical point the energy gap vanishes due to the fact that the dispersion relation of low-energy (long wavelength, small $k$ ) modes exhibits the scaling behavior $\omega_{k}=c|k|^{z}(\hbar=1)$ [23], where $c$ is a nonzero constant. We also assume that at most one quasiparticle in every mode can be excited after the quench, which is called the "single-excitation" approximation in this Letter. Then, within the adiabatic perturbation theory, the excitation probability of the $k$ th-mode quasiparticle $p_{k}$ is dominated by (assuming that there is no additional Berry phase) $[9,11,24]$

$$
p_{k} \approx\left|\int_{\lambda_{0}}^{\lambda_{1}} d \lambda\left\langle 1_{k}(\lambda)\left|\partial_{\lambda}\right| 0_{k}(\lambda)\right\rangle e^{i v^{-1} \int_{\lambda_{0}}^{\lambda} d \lambda^{\prime} \omega_{k}\left(\lambda^{\prime}\right)}\right|^{2},
$$

where $\partial_{\lambda}=\partial / \partial \lambda, \quad \lambda_{0}=\lambda\left(t_{0}\right), \quad \lambda_{1}=\lambda\left(t_{1}\right)$, and $\left|n_{k}(\lambda)\right\rangle$ denotes the instantaneous energy eigenstate of mode $k$ of $\hat{H}(\lambda)$ with the occupation number $n_{k}$. Then, the average density of excitations $\left\langle n_{\text {ex }}\right\rangle$ reads

$$
\left\langle n_{\mathrm{ex}}\right\rangle=\lim _{N \rightarrow \infty} \frac{1}{N} \sum_{k} p_{k}=\int \frac{d^{d} k}{(2 \pi)^{d}} p_{k},
$$

where $N$ denotes the number of the lattice points. In order to remove the quantity $v^{-1}$ in the exponential function in the integral of $p_{k}$ [Eq. (3)], we introduce two rescaled quantities, $\theta$ and $\phi$, defined by $[9,11,24]$

$$
\lambda=\theta v^{1 /(z \nu+1)}, \quad k=\phi v^{\nu /(z \nu+1)} .
$$

Also following Refs. [11,24], we introduce the general scaling argument

$$
\begin{aligned}
\omega_{k}(\lambda) & =|\lambda|^{z \nu} F\left(k /|\lambda|^{\nu}\right), \\
\left\langle 1_{k}^{\lambda}\left|\partial_{\lambda}\right| 0_{k}^{\lambda}\right\rangle & =\lambda^{-1} G\left(k /|\lambda|^{\nu}\right),
\end{aligned}
$$

where $F$ and $G$ are two model-dependent scaling functions satisfying $F(x) \propto x^{z}$ and $G(x) \propto x^{-1 / \nu}$ for $|x| \gg 1$. This is motivated by dimensional considerations and the requirement that the spectrum of the high energy modes should be insensitive to $\lambda$. Thus, $\left\langle n_{\mathrm{ex}}\right\rangle$ reads $[9,11]$

$$
\left\langle n_{e x}\right\rangle \approx v^{(d \nu) /(z \nu+1)} \int \frac{d^{d} \phi}{(2 \pi)^{d}} K(\phi)
$$

where

$K(\phi)=\left|\int_{\theta_{0}}^{\theta_{1}} \frac{d \theta}{\theta} G\left(\frac{\phi}{|\theta|^{\nu}}\right) \exp \left[i \int_{\theta_{0}}^{\theta} d \theta^{\prime}\left|\theta^{\prime}\right|^{z \nu} F\left(\frac{\phi}{\left|\theta^{\prime}\right|^{\nu}}\right)\right]\right|^{2}$,

with $\lambda_{0}=\theta_{0} v^{1 /(1+z \nu)}$ and $\lambda_{1}=\theta_{1} v^{1 /(1+z \nu)}$. For $d \nu /$ $(z \nu+1)<2$, the integral Eq. (8) converges in the limit $v \rightarrow 0$ and therefore $\left\langle n_{\mathrm{ex}}\right\rangle \sim v^{d \nu /(z \nu+1)}$, in accordance to the KZM prediction. But for $d \nu /(z \nu+1)>2$, the integral Eq. (8) does not converge, which means that it is not dominated by the low-energy modes [9-11]. The highenergy (ultraviolet) contribution to the integral can be 
approximated by the regular analytic adiabatic perturbation theory [9-11], which results in the quadratic scaling $\left\langle n_{\mathrm{ex}}\right\rangle \sim v^{2}$ [26]. For $d \nu /(z \nu+1)=2$, an additional logarithmic correction is expected, i.e., $\left\langle n_{\mathrm{ex}}\right\rangle \sim v^{2} \ln v$ $[10,11,33]$. This concludes our review of the KZM and the adiabatic perturbation theory. In the following, they are applied in analyzing the scaling behaviour of the work distribution during a linear quench.

Scaling behavior in the characteristic function of work.-We define the work applied during the quench on the basis of the usual two-time measurement scheme, i.e., as the difference between the results of the projective measurements [34-38] of the system's energy before and after the linear quench. It is a stochastic quantity with a distribution function $P(w)$, and the logarithm of its characteristic function [the Fourier transform of $P(w)$ ], called the cumulant $\mathrm{CFW}$, reads

$$
\begin{aligned}
\ln \chi(u) & =\ln \operatorname{Tr}\left[\hat{U}^{\dagger}\left(t_{1}, t_{0}\right) e^{i u \hat{H}\left(\lambda_{1}\right)} \hat{U}\left(t_{1}, t_{0}\right) e^{-i u \hat{H}\left(\lambda_{0}\right)} \hat{\rho}_{0}\right] \\
& =\sum_{n=1}^{\infty} \frac{(i u)^{n}}{n !} \kappa_{n},
\end{aligned}
$$

where $\hat{U}\left(t_{1}, t_{0}\right)$ is the time evolution operator, $\hat{\rho}_{0}$ is the initial state and $\kappa_{n}$ is the $n$th cumulant of work [39]. We assume that $\lambda(t)$ evolves according to the above protocol, the system is initially prepared in the ground state of $\hat{H}\left(\lambda_{0}\right)$ and the cumulant CFW satisfies a large deviation principle [40], i.e., $\lim _{N \rightarrow \infty} N^{-1} \ln \chi(u)$ exists.

For the adiabatic driving $(v \rightarrow 0)$, the system is in the ground state all the time. Hence, we have $\kappa_{1}=$ $\sum_{k}\left[\varepsilon_{k}^{0}\left(\lambda_{1}\right)-\varepsilon_{k}^{0}\left(\lambda_{0}\right)\right] \equiv N \mu$, where $\varepsilon_{k}^{0}(\lambda)$ denotes the zeropoint energy of the $k$ th mode of $\hat{H}(\lambda)$ and $\kappa_{n}=0$ for $n \geq 2$ due to the definite measurement results. Thus, the cumulant CFW for the adiabatic process should be $\ln \chi_{a}(u)=N i u \mu$ according to Eq. (9). For nonadiabatic driving ( $v$ is small but nonzero), since the nonadiabatic corrections to the cumulant CFW come from the impulse region of the KZM, we expect these corrections to exhibit the following scaling relation, i.e., $\kappa_{1}=N\left(\mu+v^{\delta_{1}} f_{1}\right)$ and $\kappa_{n}=N v^{\delta_{n}} f_{n}, n \geq 2$, where $f_{n}$ are model-dependent scaling functions and $\delta_{n}$ are the corresponding exponents characterizing the scaling behavior of each cumulant.

Every exponent $\delta_{n}$ can be determined as follows. According to Eq. (9) and in the single-excitation approximation, the scaling behavior of $\kappa_{n}$ should be the scaling behavior of $N^{-1} \sum_{k}\left[\omega_{k}\left(\lambda_{1}\right)\right]^{n} p_{k}$ because the excitations of quasiparticles in different modes are independent, i.e., $\kappa_{1} \approx N\left[\mu+N^{-1} \sum_{k} \omega_{k}\left(\lambda_{1}\right) p_{k}\right], \kappa_{n} \approx \sum_{k}\left[\omega_{k}\left(\lambda_{1}\right)\right]^{n} p_{k}$ (see Supplemental Material [26]). Now, by utilizing the expressions of $\kappa_{1}$ and $\kappa_{n}$ and following the same procedure as in the last section, we obtain

$v^{\delta_{n}} f_{n} \approx v^{(d \nu) /(z \nu+1)} \int \frac{d^{d} \phi}{(2 \pi)^{d}}\left[\omega_{k=\phi v^{\nu /(z \nu+1)}}\left(\lambda_{1}\right)\right]^{n} K(\phi)$.
If we fix $\lambda_{0}$ and $\lambda_{1}$ when varying $v$ and $\lambda_{1}$ is away from the critical point, $\omega_{k}\left(\lambda_{1}\right)$ is a nonzero constant when $k \rightarrow 0$. Also because only low-energy modes can be excited after the quench, we obtain

$$
v^{\delta_{n}} f_{n} \approx\left[\omega_{k=0}\left(\lambda_{1}\right)\right]^{n} v^{(d \nu) /(z \nu+1)} \int \frac{d^{d} \phi}{(2 \pi)^{d}} K(\phi) .
$$

Following the same analysis as that after Eq. (7), the exponents in the cumulant $\mathrm{CFW}$ read

$$
v^{\delta_{n}}= \begin{cases}v^{d \nu /(z \nu+1)} & d \nu /(z \nu+1)<2, \\ v^{2} \ln v & d \nu /(z \nu+1)=2, \\ v^{2} & d \nu /(z \nu+1)>2\end{cases}
$$

Finally, according to Eq. (9), since in this case $\delta_{n}$ is independent of $n, \ln \chi(u)$ reads

$$
\ln \chi(u)=\ln \chi_{a}(u)+N v^{\delta_{n}} f(i u),
$$

where $f(i u) \equiv \sum_{n=1}^{\infty}(i u)^{n} f_{n} / n$ !. We would like to emphasize that the scaling behavior exists not only in the CFW but also in the work distribution [see Eq. (14)]. According to the Gartner-Ellis theorem, the distribution of the work per lattice site $P_{N}(w) \equiv P(N w)$ also takes on the large deviation form, $\lim _{N \rightarrow \infty} N^{-1} P_{N}(w)=-I(w)$. Here, the rate function $I(w)$ is obtained by the Legendre-Fenchel transform [40] via

$$
I(w)=\sup _{u \in \mathbb{R}}\left\{u(w-\mu)-v^{\delta_{n}} f(u)\right\} .
$$

Now, let us consider a second case: $\lambda_{1}$ is near the critical point. Since in this case $\omega_{k}\left(\lambda_{1}\right)=c|k|^{z}$ when $k \rightarrow 0$, according to Eq. (10), we have

$$
v^{\delta_{n}} f_{n} \approx c^{n} v^{[(d+n z) \nu] /[z \nu+1]} \int \frac{d^{d} \phi}{(2 \pi)^{d}}|\phi|^{n z} K(\phi) .
$$

Similar to the discussion about $\left\langle n_{\mathrm{ex}}\right\rangle$, we obtain

$$
v^{\delta_{n}}= \begin{cases}v^{(d+n z) \nu /(z \nu+1)} & (d+n z) \nu /(z \nu+1)<2, \\ v^{2} \ln v & (d+n z) \nu /(z \nu+1)=2, \\ v^{2} & (d+n z) \nu /(z \nu+1)>2 .\end{cases}
$$

We note that the quantity in Eq. (15) for $n=1$ is called the excess energy in Refs. [10,11]. If $\delta_{1}<2$, to a good approximation, we can cut off the sum in Eq. (9) to the first order $(n=1)$ for sufficiently small $v$ and obtain

$$
\ln \chi(u) \approx \ln \chi_{a}(u)+N v^{\delta_{1}} i u f_{1} .
$$

Accordingly, $P_{N}(w)$ is a Dirac delta distribution located at $\mu+v^{\delta_{1}} f_{1}$. 

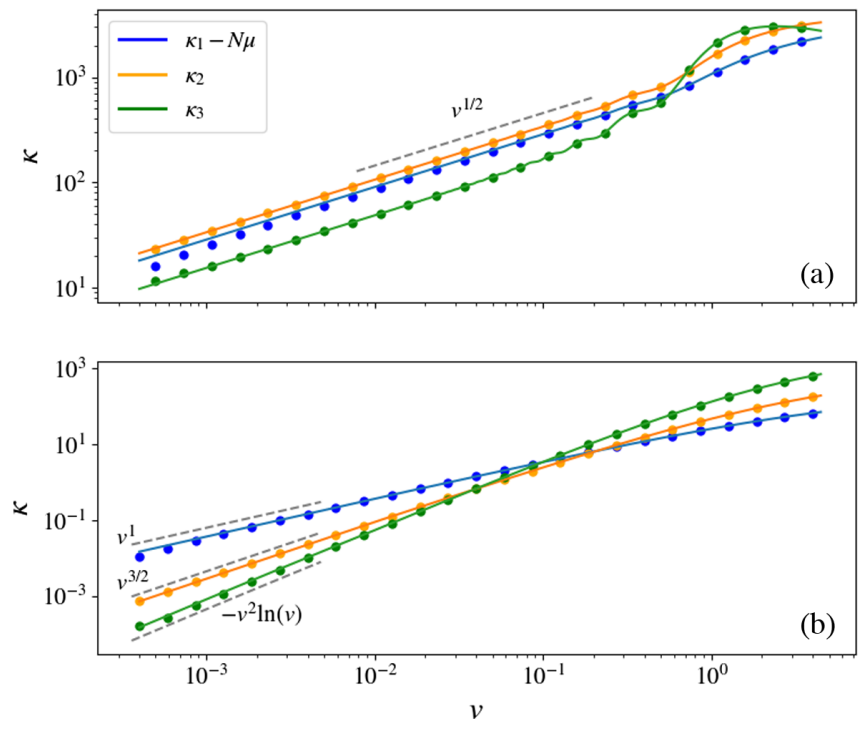

FIG. 1. The first three cumulants of the work distribution as a function of the quenching rate for a 1D transverse Ising chain. Solid lines correspond to the exact analytic solution in the macroscopic limit [42] and dots to an exact numerical simulation of a chain of $N$ spins. (a) Quench between $\lambda_{0}=-4$ and $\lambda_{1}=1$, for which all the exponents are $1 / 2$. In this case we have $N=$ 4000 and the deviations at low $v$ are due to the finite size effects [12]. (b) Quench between $\lambda_{0}=-4$ and $\lambda_{1}=0$, for which our theory predicts $\kappa_{1}-N \mu \propto v, \kappa_{2} \propto v^{3 / 2}$ and $\kappa_{3} \propto v^{2} \ln v$. Even in this case of a shorter chain $(N=1000)$, finite size effects are less relevant. This is natural given the scale invariance at the critical point, which is the ending point of the protocol.

In summary, our analysis shows that the scaling of the work cumulants is qualitatively different depending on whether $\lambda_{1}=0$. If $\lambda_{1} \neq 0$, all the cumulants (for whatever $n$ ) have the same scaling exponent, while if $\lambda_{1}=0$, they do not. This is illustrated in Fig. 1 by the exact numerical simulation of the 1D transverse Ising model [41], which is also studied analytically in the following. It is important to note that this difference between the two kinds of quenches is not observed for the density of excitations $n_{\mathrm{ex}}$, which displays the same scaling behavior irrelevant to the ending point of the protocol.

Example.-We calculate the CFW of the 1D transverse Ising model to demonstrate our results since it is solvable and the KZM is valid in this model [25,41]. The Hamiltonian of a chain of $N$ spins in a transverse magnetic field reads

$$
\hat{H}(\lambda)=-J \sum_{l=1}^{N}\left[\hat{\sigma}_{l}^{z} \hat{\sigma}_{l+1}^{z}+(\lambda-1) \hat{\sigma}_{l}^{x}\right],
$$

with the Born-von Kármán periodic boundary condition. Here, $\hat{\sigma}_{l}^{x, y, z}$ denote the Pauli matrices on site $l$, and $J$ denotes the energy scale. The critical points are at $\lambda=0,2$. Moreover, $d=z=\nu=1[24,25]$. For the critical point $\lambda=0$, we choose $\lambda(t)=v t, \lambda_{0}<0$ and $0<\lambda_{1}<2$.
According to Ref. [45], when $N \rightarrow \infty$, the cumulant CFW reads

$$
\ln \chi(u)=\frac{N}{\pi} \int_{0}^{\pi} d k \ln \frac{g_{k}(u)}{g_{k}(0)},
$$

where

$$
\begin{aligned}
g_{k}(u)= & \left\{1+\cos \left(u \omega_{k}^{1}\right) \cos \left[(u-i \beta) \omega_{k}^{0}\right]\right. \\
& \left.+Q_{k} \sin \left(u \omega_{k}^{1}\right) \sin \left[(u-i \beta) \omega_{k}^{0}\right]\right\}^{1 / 2} .
\end{aligned}
$$

Here, $\beta=\left(k_{B} T\right)^{-1}$ is the inverse temperature of the canonical initial state, $\omega_{k}^{0}=\omega_{k}\left(\lambda_{0}\right), \omega_{k}^{1}=\omega_{k}\left(\lambda_{1}\right)$, where $\omega_{k}(\lambda)=2 J \sqrt{(\lambda-1+\cos k)^{2}+\sin ^{2} k}$ is the energy of the $k$ th mode. Also, $Q_{k}=1-2 p_{k}$, where $p_{k} \approx e^{-2 \pi J k^{2} / v}$ is the excitation probability in the corresponding Landau-Zener model for mode $k$ [41]. From Eq. (19), we obtain the first and the second cumulants of work

$$
\begin{aligned}
\kappa_{1}= & \frac{N}{2 \pi} \int_{0}^{\pi} d k\left(\omega_{k}^{0}-Q_{k} \omega_{k}^{1}\right) \tanh \left(\frac{\beta \omega_{k}^{0}}{2}\right) \\
\kappa_{2}= & \frac{N}{4 \pi} \int_{0}^{\pi} d k\left[\left(\omega_{k}^{0}-Q_{k} \omega_{k}^{1}\right)^{2}+\left(1-Q_{k}^{2}\right)\left(\omega_{k}^{1}\right)^{2} \cosh \left(\beta \omega_{k}^{0}\right)\right] \\
& \times \operatorname{sech}^{2}\left(\frac{\beta \omega_{k}^{0}}{2}\right) .
\end{aligned}
$$

Quantum phase transitions occur at the absolute zero. Hence, we consider that case in which the initial state is chosen to be the ground state of $\hat{H}\left(\lambda_{0}\right)$. From Eq. (19), we have

$$
\begin{aligned}
\ln \chi(u) & =N \int_{0}^{\pi} \frac{d k}{2 \pi}\left\{i u\left(\omega_{k}^{0}-\omega_{k}^{1}\right)+\ln \left[1+p_{k}\left(e^{2 i u \omega_{k}^{1}}-1\right)\right]\right\}, \\
& =\ln \chi_{a}(u)+N \sum_{n=1}^{\infty} \frac{(-1)^{n+1}}{n} \int_{0}^{\pi} \frac{d k}{2 \pi} p_{k}^{n}\left(e^{2 i u \omega_{k}^{1}}-1\right)^{n},
\end{aligned}
$$

where $\ln \chi_{a}(u)=\operatorname{Niu\mu }=\operatorname{Niu}(2 \pi)^{-1} \int_{0}^{\pi} d k\left(\omega_{k}^{0}-\omega_{k}^{1}\right)$ is the cumulant $\mathrm{CFW}$ for the adiabatic process. The sum in the last equation is convergent under the condition $\left|p_{k}\left(e^{2 i u \omega_{k}^{1}}-1\right)\right|=2 p_{k}\left|\sin \left(u \omega_{k}^{1}\right)\right|<1$ [46]. Also, for $T=0$, from Eq. (22), we have

$$
\begin{aligned}
& \kappa_{1}=N\left(\mu+\frac{1}{\pi} \int_{0}^{\pi} d k \omega_{k}^{1} p_{k}\right), \\
& \kappa_{2}=\frac{2 N}{\pi} \int_{0}^{\pi} d k\left(\omega_{k}^{1}\right)^{2} p_{k}\left(1-p_{k}\right), \\
& \kappa_{3}=\frac{4 N}{\pi} \int_{0}^{\pi} d k\left(\omega_{k}^{1}\right)^{3} p_{k}\left(1-p_{k}\right)\left(1-2 p_{k}\right) .
\end{aligned}
$$

Due to the exponential decay of $p_{k}$, only low-energy modes can get excited. Thus, we extend the upper limit of the 
integral to $\infty$ and we approximately have $p_{k} e^{2 i u \omega_{k}^{1}} \approx$ $p_{k} e^{2 i u \omega_{k=0}^{1}}=p_{k} e^{4 i u J \lambda_{1}}$. In this way, from Eqs. (22) and (23), we obtain

$$
\begin{aligned}
\frac{1}{N} \ln \frac{\chi(u)}{\chi_{a}(u)} & =\sum_{n=1}^{\infty} \frac{(-1)^{n+1}}{n} \frac{\left(e^{4 i u J \lambda_{1}}-1\right)^{n}}{2 \pi} \int_{0}^{\infty} d k e^{-2 n \pi J k^{2} / v}, \\
& =\frac{-v^{1 / 2} J^{-1 / 2} \sqrt{2} \mathrm{Li}_{3 / 2}\left(1-e^{4 i u J \lambda_{1}}\right)}{8 \pi}
\end{aligned}
$$

and

$$
\begin{aligned}
& \kappa_{1}=N\left(\mu+\frac{v^{1 / 2} J^{1 / 2} \lambda_{1} \sqrt{2}}{2 \pi}\right), \\
& \kappa_{2}=N \frac{v^{1 / 2} J^{3 / 2} \lambda_{1}^{2}(2 \sqrt{2}-2)}{\pi}, \\
& \kappa_{3}=N \frac{v^{1 / 2} J^{5 / 2} \lambda_{1}^{3} 4(6 \sqrt{2}-18+4 \sqrt{6})}{3 \pi},
\end{aligned}
$$

where $\operatorname{Li}_{s}(z)=\sum_{l=1}^{\infty} z^{l} / l^{s}$ is the polylogarithm function. Also, we have $f(u)=-\sqrt{2 / J} \operatorname{Li}_{3 / 2}\left(1-e^{4 J u \lambda_{1}}\right) /(8 \pi)$ and $\delta_{n}=1 / 2=d \nu /(z \nu+1)$. Obviously, the 1D transverse Ising model verifies our predictions in Eqs. (12) and (13).

We also would like to do some quantitative analysis about the work distribution function. $f(u)$ is a monotonic function with the following asymptotic behavior: for $u \rightarrow-\infty, f(u)=-\sqrt{2 / J} \zeta(3 / 2) /(8 \pi)$, where $\zeta(z)$ is the Riemann zeta function; for $u \rightarrow \infty$ [the domain of $f(u)$ has been extended to the real axis by applying analytic continuation], $f(u)=J\left(\lambda_{1} u\right)^{3 / 2} \sqrt{2} /[\pi \Gamma(5 / 2)]$, where $\Gamma(z)$ is the Gamma function. Hence, from the asymptotic behavior of $f(u)$ and by applying the Legendre-Fenchel transform, we find that for $w<\mu, P_{N}(w)=0$ which is consistent with the initial ground state condition. A confusion may arise when we consider $w>w_{m} \equiv \mu+$ $\int_{0}^{\pi} d k \omega_{k}^{1} / \pi$ since now $P_{N}(w)$ is the probability of unphysical events. This is a consequence of the approximation in which we extend the upper limit of the integral to $\infty$. Actually, it can be proved that when $w>w_{m}$, $I(w)>I\left(w_{m}\right) \propto v^{-1} . \quad$ Because $\quad P_{N}(w) \propto e^{-N I(w)}$, $P_{N}(w)<P_{N}\left(w_{m}\right) \ll 1$, which indicates that the probabilities of the unphysical events are sufficiently small and our approximation is still reasonable.

If $\lambda_{1}$ is near the critical point, $\omega_{k}^{1}=2 J k$ when $k \rightarrow 0$. And for every mode, the dynamics corresponds to a half Landau-Zener problem $[11,24,43,44]$, where $p_{k}$ reads

$$
\begin{aligned}
p_{k}=1 & -2 \frac{e^{-\pi \alpha_{k} / 8}}{\pi \alpha_{k}} \sinh \left(\frac{\pi \alpha_{k}}{4}\right) \\
& \times\left|\Gamma\left(1+\frac{i \alpha_{k}}{8}\right)+\sqrt{\frac{\alpha_{k}}{8}} \Gamma\left(\frac{1}{2}+\frac{i \alpha_{k}}{8}\right) e^{i \pi / 4}\right|^{2},
\end{aligned}
$$

with $\alpha_{k}=4 J k^{2} / v$. This function has the following asymptotic behavior: for $\alpha_{k} \rightarrow 0, p_{k}=1 / 2$; for $\alpha_{k} \rightarrow \infty$, $p_{k}=1 /\left(2 \alpha_{k}\right)^{2}$. Because only low-energy modes can get excited after the quench, we have

$\kappa_{1}=N\left(\mu+\frac{2 J}{\pi} \int_{0}^{\infty} d k k p_{k}\right) \approx N(\mu+0.038 v)$,

$\kappa_{2}=\frac{8 N J^{2}}{\pi} \int_{0}^{\infty} d k k^{2} p_{k}\left(1-p_{k}\right) \approx 0.092 N v^{3 / 2} J^{1 / 2}$.

For $n \geq 3$, the upper limit of the integral cannot be extended to $\infty$ due to the power-law decay of $p_{k}$. After some careful analysis, we find for $n>3, \kappa_{n} \sim v^{2}$ is reproduced [47]. Moreover, for $n=3$, the logarithmic correction appears: $\kappa_{3} \sim v^{2} \ln v$. These results again verify our predictions in Eq. (16).

Conclusions. - In this Letter, we have studied the statistics of the work applied across a quantum phase transition in systems characterized by independent quasiparticles. We have shown that all the cumulants of the work distribution exhibit a scaling behavior for small quench rates, and that the scaling exponents are determined by the dimension of the system and the critical exponents of the transition. This is in analogy to the predictions of the KZM, although there are qualitative differences in quenches ending close to and away from the critical point. In addition, we are also able to determine the scaling exponents $\delta_{n}$ when (1) the energy spectrum is always gapped during the protocol, (2) the initial state is not the ground state, or (3) the protocol is a sudden quench protocol near the critical point (see the Supplemental Material [26]). We also show that although the cumulant CFW for slow linear quenches traversing a critical point is analytic for $u \simeq 0$ (which allows to properly define the cumulants), it has nonanalyticities at certain values of $u$. This is related to the phenomenon of dynamical quantum phase transitions [26], which has been previously reported for the case of sudden quenches $[18,19,48]$.

H. T. Q. gratefully acknowledges support from the National Science Foundation of China under Grants No. 11775001, No. 11534002, and No. 11825001. N.F. and M.E. acknowledge funding from the European Research Council project NanoThermo (ERC-2015-CoG Agreement No. 681456). V. C. is funded by the National Research Fund of Luxembourg in the frame of Project No. QUTHERM C18/MS/12704391.

*htquan@pku.edu.cn †massimiliano.esposito@uni.lu

[1] T. W. B. Kibble, J. Phys. A 9, 1387 (1976).

[2] T. W. B. Kibble, Phys. Rep. 67, 183 (1980).

[3] W. H. Zurek, Nature (London) 317, 505 (1985).

[4] W. H. Zurek, Phys. Rep. 276, 177 (1996). 
[5] T. Albash and D. A. Lidar, Rev. Mod. Phys. 90, 015002 (2018).

[6] K. Kim, M.-S. Chang, S. Korenblit, R. Islam, E. E. Edwards, J. K. Freericks, G.-D. Lin, L.-M. Duan, and C. Monroe, Nature (London) 465, 590 (2010).

[7] J. D. Biamonte, V. Bergholm, J. D. Whitfield, J. Fitzsimons, and A. Aspuru-Guzik, AIP Adv. 1, 022126 (2011).

[8] S. Deffner, Phys. Rev. E 96, 052125 (2017).

[9] A. Polkovnikov, Phys. Rev. B 72, 161201(R) (2005).

[10] A. Polkovnikov, K. Sengupta, A. Silva, and M. Vengalattore, Rev. Mod. Phys. 83, 863 (2011).

[11] C. De Grandi and A. Polkovnikov, Quantum Quenching, Annealing and Computation (Springer, Berlin, Heidelberg, 2010), pp. 75-114.

[12] A. delCampo, Phys. Rev. Lett. 121, 200601 (2018).

[13] J. M. Cui, F. J. Gómez-Ruiz, Y. F. Huang, C. F. Li, G. C. Guo, and A. D. Campo, Commun. Phys. 3, 44 (2020); Y. Bando et al., arXiv:2001.11637.

[14] K. Sekimoto, Stochastic Energetics (Springer-Verlag, Berlin, Heidelberg, 2010), https://link.springer.com/book/10 .1007\%2F978-3-642-05411-2.

[15] C. Jarzynski, Annu. Rev. Condens. Matter Phys. 2, 329 (2011).

[16] U. Seifert, Rep. Prog. Phys. 75, 126001 (2012).

[17] R. Dorner, J. Goold, C. Cormick, M. Paternostro, and V. Vedral, Phys. Rev. Lett. 109, 160601 (2012).

[18] A. Silva, Phys. Rev. Lett. 101, 120603 (2008).

[19] M. Heyl, A. Polkovnikov, and S. Kehrein, Phys. Rev. Lett. 110, 135704 (2013).

[20] H. T. Quan, Z. Song, X. F. Liu, P. Zanardi, and C. P. Sun, Phys. Rev. Lett. 96, 140604 (2006).

[21] B. Damski, H. T. Quan, and W. H. Zurek, Phys. Rev. A 83, 062104 (2011).

[22] B. Dóra, Á. Bácsi, and G. Zaránd, Phys. Rev. B 86, 161109 (R) (2012).

[23] B. I. Halperin, Phys. Today 72, No. 242 (2019).

[24] J. Dziarmaga, Adv. Phys. 59, 1063 (2010).

[25] W. H. Zurek, U. Dorner, and P. Zoller, Phys. Rev. Lett. 95, 105701 (2005).

[26] See the Supplemental Material at http://link.aps.org/ supplemental/10.1103/PhysRevLett.124.170603 for extensions of the main results, the derivation of the $\mathrm{CFW}$ for systems with independent quasiparticles, and connections to dynamical phase transitions, which includes Refs. [27-32].
[27] C. De Grandi, V. Gritsev, and A. Polkovnikov, Phys. Rev. B 81, 012303 (2010).

[28] C. De Grandi, V. Gritsev, and A. Polkovnikov, Phys. Rev. B 81, 224301 (2010).

[29] B. Damski, Phys. Rev. E 87, 052131 (2013).

[30] C. P. Sun, J. Phys. A 21, 1595 (1988).

[31] G. Rigolin, G. Ortiz, and V. H. Ponce, Phys. Rev. A 78, 052508 (2008).

[32] P. Talkner, P. S. Burada, and P. Hänggi, Phys. Rev. E 78, 011115 (2008).

[33] S. J. Gu, Int. J. Mod. Phys. B 24, 4371 (2010).

[34] J. Kurchan, arXiv:cond-mat/0007360.

[35] H. Tasaki, arXiv:cond-mat/0009244.

[36] P. Talkner, E. Lutz, and P. Hänggi, Phys. Rev. E 75, 050102 (R) (2007).

[37] M. Esposito, U. Harbola, and S. Mukamel, Rev. Mod. Phys. 81, 1665 (2009).

[38] M. Campisi, P. Hänggi, and P. Talkner, Rev. Mod. Phys. 83, 771 (2011).

[39] For $n \leq 3, \kappa_{n}$ is equal to the $n$th central moment of work.

[40] H. Touchette, Phys. Rep. 478, 1 (2009).

[41] J. Dziarmaga, Phys. Rev. Lett. 95, 245701 (2005).

[42] The analytical results in the macroscopic limit are obtained by evaluating the integrals in Eq. (23). Here, the probabilities $p_{k}$ are from the exact solution of the Landau-Zener problem $[43,44]$. Thus, the results are valid for arbitrary quench rates.

[43] N. V. Vitanov, Phys. Rev. A 59, 988 (1999).

[44] B. Damski and W. H. Zurek, Phys. Rev. A 73, 063405 (2006).

[45] Z. Y. Fei and H. T. Quan, Phys. Rev. Research 1, 033175 (2019).

[46] The condition for the convergence of the series $2 p_{k}\left|\sin \left(u \omega_{k}^{1}\right)\right|<1$ is always satisfied in Eq. (22) when we calculate the cumulants of work, because $u \rightarrow 0$ in this case.

[47] In fact, for $n>3$, Eq. (26) overestimates the results compared with numerical results because for high-energy modes, the calculation of $p_{k}$ does not exactly correspond to a half Landau-Zener problem. However, Eq. (26) does lead to the correct scaling behavior of the cumulant CFW.

[48] N. O. Abeling and S. Kehrein, Phys. Rev. B 93, 104302 (2016). 


\title{
Supplemental Material: Work statistics across a quantum phase transition
}

\author{
Zhaoyu Fei, ${ }^{1}$ Nahuel Freitas, ${ }^{2}$ Vasco Cavina, ${ }^{2}$ H. T. Quan,,${ }^{1,4}$ and Massimiliano Esposito ${ }^{2}$ \\ ${ }^{1}$ School of Physics, Peking University, Beijing 100871, China \\ ${ }^{2}$ Complex Systems and Statistical Mechanics, Physics and Materials Science, \\ University of Luxembourg, L-1511 Luxembourg, Luxembourg \\ ${ }^{3}$ Collaborative Innovation Center of Quantum Matter, Beijing 100871, China \\ ${ }^{4}$ Frontiers Science Center for Nano-optoelectronics, Peking University, Beijing, 100871, China
}

\section{A: the regular analytic adiabatic perturbation theory}

If the energy spectrum is always gapped during the protocol, the scaling of the number of excitations can be obtained by applying the regular adiabatic perturbation theory. According to Refs. [1-3], for a linear quench $\lambda(t)=\lambda_{0}+v\left(t-t_{0}\right)$ with a sufficiently small $v$, the transition probability $P_{m, n}$ from the $m$ th instantaneous eigenstate $\left|E_{m}^{\lambda_{0}}\right\rangle$ with energy $E_{m}^{\lambda_{0}}$ to the $n$th instantaneous eigenstate $\left|E_{n}^{\lambda_{1}}\right\rangle$ with energy $E_{n}^{\lambda_{1}}(m \neq n)$ reads

$$
P_{m, n}=v^{2}\left[\frac{\left|\left\langle E_{n}^{\lambda_{0}}\left|\partial_{\lambda_{0}}\right| E_{m}^{\lambda_{0}}\right\rangle\right|^{2}}{\left(E_{n}^{\lambda_{0}}-E_{m}^{\lambda_{0}}\right)^{2}}+\frac{\left|\left\langle E_{n}^{\lambda_{1}}\left|\partial_{\lambda_{1}}\right| E_{m}^{\lambda_{1}}\right\rangle\right|^{2}}{\left(E_{n}^{\lambda_{1}}-E_{m}^{\lambda_{1}}\right)^{2}}-2 \cos \left(\gamma_{m n}\right) \frac{\left\langle E_{n}^{\lambda_{0}}\left|\partial_{\lambda_{0}}\right| E_{m}^{\lambda_{0}}\right\rangle}{E_{n}^{\lambda_{0}}-E_{m}^{\lambda_{0}}} \frac{\left\langle E_{n}^{\lambda_{1}}\left|\partial_{\lambda_{1}}\right| E_{m}^{\lambda_{1}}\right\rangle}{E_{n}^{\lambda_{1}}-E_{m}^{\lambda_{1}}}\right]
$$

where, without considering the Berry phase, we have $\gamma_{m n}=v^{-1} \int_{\lambda_{0}}^{\lambda_{1}} d \lambda\left[E_{m}(\lambda)-E_{n}(\lambda)\right]$, and in order to obtain a meaningful expansion, we require $v\left\langle E_{n}^{\lambda}\left|\partial_{\lambda}\right| E_{m}^{\lambda}\right\rangle \ll E_{n}^{\lambda}-E_{m}^{\lambda}$ for any $n \neq m$ and $\lambda$. Eq. (S1) indicates that the probability of excitation is proportional to $v^{2}$ (we ignored the highly oscillating cosine function in Eq. (S1)). From this result, we can conclude, following the reasoning of Sec. III of the main text, that the scaling exponents in the CFW are $\delta_{n}=2, \forall n$. Moreover for later convenience, we also consider a sudden quench protocol with a sufficiently small amplitude $v$

$$
\lambda(t)=\lambda_{0}+v \Theta(t)
$$

where $\Theta(t)$ is the Heaviside step function. As shown in Refs. [3-5], we have

$$
P_{m, n}=v^{2}\left|\left\langle E_{n}^{\lambda_{0}}\left|\partial_{\lambda_{0}}\right| E_{m}^{\lambda_{0}}\right\rangle\right|^{2}
$$

and in this case we also obtain $\delta_{n}=2$.

We demonstrate these results by using the analytic results of the cumulant CFW of a forced harmonic oscillator, with the Hamiltonian given by

$$
\hat{H}(\lambda)=\frac{\hat{p}^{2}}{2 m}+\frac{1}{2} m \omega^{2} \hat{x}^{2}+\lambda \sqrt{\frac{2 m \omega}{\hbar}} \hat{x} .
$$

According to Ref. [6], the CFW of this model satisfies the following equation

$$
\ln \frac{\chi(u)}{\chi_{a}(u)}=\left[\left(e^{i u \hbar \omega}-1\right)-\frac{4 \sin ^{2}(u \hbar \omega / 2)}{e^{\beta \hbar \omega}-1}\right] A(\omega),
$$

where $A(\omega)=\left|\int_{t_{0}}^{t_{1}} \mathrm{~d} s \dot{\lambda}(s) e^{i \omega s}\right|^{2}$. It is easy to check that for the linear quench protocol,

$$
A(\omega)=\frac{2 v^{2}}{w^{2}}\left\{1-\cos \left[\frac{\left(\lambda_{1}-\lambda_{0}\right) \omega}{v}\right]\right\}
$$

and for the sudden quench protocol, $A(\omega)=v^{2}$, confirming in this way the quadratic scaling of the cumulant $\mathrm{CFW}$ with respect to $v$ for both protocols.

\section{B: the CFW for the system characterized by independent quasiparticles}

For independent excitations of quasiparticles, the work performed during the quench process reads $w=N \mu+$ $\sum_{k} \omega_{k} n_{k}$, where $n_{k}$ denotes the occupation number of the $k$ th mode at the end of the process. Let us use $p_{n_{k}}$ to 
denote the discrete probability distribution of $n_{k}$ after the quench. Since $w$ is the sum of the independent random variables $\omega_{k} n_{k}$, by additivity of the cumulant CFW, we can straightforwardly obtain

$$
\ln \chi(u)=N i u \mu+\sum_{k} \ln \chi_{k}(u)
$$

where $\chi_{k}(u)=\sum_{n_{k}} e^{i u \omega_{k} n_{k}} p_{n_{k}}$ is the characteristic function of $\omega_{k} n_{k}$. Notice that $p_{n_{k}}$ depends on the work parameter $\lambda$, like the other relevant quantities $\mu$ and $\omega_{k}$. Now, due to the single-excitation approximation, only the configurations in which at most one quasiparticle in each mode gets excited at a time will be counted. Hence, for the excited mode $k$, we let $p_{n_{k}=1} \approx p_{k}, p_{n_{k}=0} \approx 1-p_{k}$ and $p_{n_{k}} \approx 0$ for $n_{k} \geq 2$. Thus, the random variables $n_{k}$ are independent Bernoulli trials and the trajectory work $w=N \mu+\sum_{k} \omega_{k} n_{k}$ is the linear combination of independent Bernoulli trials. Computing the CFW by adding up the contributions from all the configurations described above, we have

$$
\ln \chi(u) \approx N i u \mu+\sum_{k} \ln \left[1+p_{k}\left(e^{i u \omega_{k}}-1\right)\right] .
$$

In another word, the trajectory work satisfies a "modified" Poisson binomial distribution.

Hence, according to Eq. (9), we obtain the cumulants of work $\kappa_{1} \approx N \mu+\sum_{k} \omega_{k} p_{k}$ and $\kappa_{n} \approx \sum_{k} \omega_{k}^{n} p_{k}$. Here we have ignored the quantity $\left(p_{k}\right)^{m}$ for $m \geq 2$, which does not influence the scaling behavior of the CFW.

\section{C: the exponents in the CFW for an initial state other than the ground state}

It is interesting to note that the cumulant CFW also exhibit scaling behavior when the initial state is not the ground state. In the following, we will discuss three cases corresponding to three different initial states:

(i) We consider an arbitrary excited state as the initial state: The scaling relations given by Eq. (1) are the consequence of the vanishing energy gap (due to the excitation energies of the low-energy modes approach zero) at the quantum critical point. With this in mind, the critical behavior is independent of the choice of the initial state and is expected to hold also when initializing our quantum system in an excited configuration. Since the adiabatic-impulse-adiabatic approximation in KZM and the adiabatic perturbation theory are still valid in this case, we can apply the same reasoning in sections II and III of the main text to find that $\delta_{n}$ are exactly the same as the ones for the initial ground state.

(ii) For the canonical initial state at a finite temperature $T$, the above results are also valid when $\lambda_{0}$ is sufficiently far from the critical point. The reason can be simply understood as follows. The canonical state is a mixture of energy eigenstates. Since for every energy eigenstate, the cumulant CFW exhibits the same scaling behavior and the coefficients of these eigenstates are irrelevant to the scaling behavior, the mixture of these energy eigenstates certainly leads to the same scaling behavior as the ones for the initial ground state.

(iii) For the canonical initial state, when the temperature $T$ is high and $\lambda_{0}$ is near the critical point, the coefficients of these eigenstates are relevant to the scaling behavior. As a result, the cumulant CFW exhibits a different scaling behavior. Specifically, $v^{\delta_{n}}$ can achieve larger (for bosons) or smaller (for fermions) values than the ones derived in the main text [3].

In following, we will demonstrate results of case (ii) and case (iii) with the 1D transverse Ising model initially prepared in a canonical distribution.

For case (ii), both $\lambda_{0}$ and $\lambda_{1}$ are away from the critical point, due to the exponential decay of $p_{k}$, only low-energy modes can get excited after the quench, we have $p_{k} h\left(\omega_{k}(\lambda)\right) \approx p_{k} h\left(\omega_{k=0}(\lambda)\right)$, where $h\left(\omega_{k}(\lambda)\right)$ is an arbitrary smooth function of $\omega_{k}(\lambda)$. Also, according to Eqs. $(19,20)$, we have

$$
\ln \chi_{a}(u)=\frac{N}{2 \pi} \int_{0}^{\pi} \mathrm{d} k \ln \frac{1+\cos \left(u \omega_{k}^{1}-u \omega_{k}^{0}+i \beta \omega_{k}^{0}\right)}{1+\cosh \left(\beta \omega_{k}^{0}\right)},
$$

where $\ln \chi_{a}(u)$ is the cumulant CFW under the adiabatic driving. Then from Eqs. (19, 20, 21), we obtain

$$
\begin{aligned}
\frac{1}{N} \ln \frac{\chi(u)}{\chi_{a}(u)} & =\frac{-v^{1 / 2} J^{-1 / 2} \sqrt{2}}{8 \pi} \operatorname{Li}_{3 / 2}\left(\frac{-2 \sin \left(2 J \lambda_{1} u\right) \sin \left[2 J \lambda_{0}(u-i \beta)\right]}{1+\cos \left[2 J\left(\lambda_{1} u+\lambda_{0} u-i \lambda_{0} \beta\right)\right]}\right), \\
\kappa_{1} & =N \mu+N \frac{v^{1 / 2} J^{1 / 2} \lambda_{1} \sqrt{2}}{2 \pi} \tanh \left(\beta J \lambda_{0}\right), \\
\kappa_{2} & =\kappa_{2 a}+N \frac{v^{1 / 2} J^{3 / 2} \lambda_{1}\left[(2 \sqrt{2}-2) \lambda_{1} \sinh ^{2}\left(\beta J \lambda_{0}\right)-\sqrt{2} \lambda_{0}\right]}{\pi \cosh ^{2}\left(\beta J \lambda_{0}\right)},
\end{aligned}
$$


where the first and the second cumulants of work under the adiabatic driving are given by

$$
\begin{aligned}
\mu & =\frac{1}{2 \pi} \int_{0}^{\pi} \mathrm{d} k\left(\omega_{k}^{0}-\omega_{k}^{1}\right) \tanh \left(\frac{\beta \omega_{k}^{0}}{2}\right), \\
\kappa_{2 a} & =\frac{N}{4 \pi} \int_{0}^{\pi} \mathrm{d} k\left(\omega_{k}^{0}-\omega_{k}^{1}\right)^{2} \operatorname{sech}^{2}\left(\frac{\beta \omega_{k}^{0}}{2}\right) .
\end{aligned}
$$

It can be seen from Eq. (S10), the exponents are $\delta_{n}=1 / 2$, just the same as that for the initial ground state (Eqs. (24, 25)).

For case (iii), $\lambda_{0}$ is near the critical point and $\lambda_{1}$ is away from it, $\omega_{k}^{0}=2 J k$ when $k \rightarrow 0$ and $p_{k}$ is given by Eq. (26) because we fall again in the half Landau-Zener scenario $[3,7,8]$. Thus, in the high temperature limit, since only low-energy modes can get excited after the quench, from Eqs. (19, 20, 21), we have

$$
\begin{aligned}
\frac{1}{N} \ln \frac{\chi(u)}{\chi_{a}(u)} & =\frac{2 J}{\pi}(i \beta-u) \tan \left(J \lambda_{1} u\right) \int_{0}^{\infty} \mathrm{d} k k p_{k}=0.038 v(i \beta-u) \tan \left(J \lambda_{1} u\right) \\
\kappa_{1} & =N\left(\mu+0.038 \beta v J \lambda_{1}\right) \\
\kappa_{2} & =\kappa_{2 a}+0.076 N v J \lambda_{1} .
\end{aligned}
$$

Comparing Eq. (S12) with Eq. (S10), we recognize that the scaling $v^{1 / 2}$ becomes $\beta v$ for the odd-order cumulants of work and $v$ for the even-order cumulants of work. This is compatible with the fermionic anti-bunching effects discussed in Ref. [3], since the quasiparticles in the transverse Ising model are fermions.

\section{D: sudden quench protocol near the critical point}

In this section, we discuss the scaling behavior of the cumulant $\mathrm{CFW}$ for the sudden quench protocol near the critical point

$$
\lambda(t)=v \Theta(t)
$$

If the quench amplitude $v$ is sufficiently small, the adiabatic perturbation theory is still valid [3-5] and we can write

$$
p_{k} \approx\left|\int_{0}^{v} \frac{\mathrm{d} \lambda}{\lambda} G\left(\frac{k}{\lambda^{\nu}}\right)\right|^{2}
$$

where $G(x)$ is the scaling function defined in Eq. (6) of the main text. To get rid of the dependence on $v$ in the upper limit of the integral, we can define the following rescaled quantities: $\lambda=\theta v, k=\phi v^{\nu}$ and finally obtain, from Eq. (9) of the main text,

$$
v^{\delta_{n}}=\left\{\begin{array}{lc}
v^{(d+n z) \nu} & (d+n z) \nu<2 \\
v^{2} \ln v & (d+n z) \nu=2 \\
v^{2} & (d+n z) \nu>2
\end{array}\right.
$$

in which the exponents explicitly depend on $n$. Please note that results in Eq. (S15) are different from those in Eq. (S3).

Again, we use the 1D transverse Ising model to demonstrate our results. According to Ref. [9], $p_{k}$ is such that

$$
1-2 p_{k}=\frac{(v-1+\cos k)(-1+\cos k)+\sin ^{2} k}{\sqrt{\left[(v-1+\cos k)^{2}+\sin ^{2} k\right]\left[(-1+\cos k)^{2}+\sin ^{2} k\right]}} .
$$

Although $v$ is small, we cannot directly expand the equation above in Taylor series since it will result in a divergence of the excitation probability for $k=0$ and make the integral in Eq. (23) ill-defined. This also happens to the fidelity susceptibility at the critical point of a transverse Ising model [10, 11]. To obtain the proper integral, we have to first rescale $k=k^{\prime} v$ and then perform the series expansion. With this in mind, for low energy modes, we have

$$
p_{k} \approx \frac{\sqrt{1+k^{\prime 2}}-k^{\prime}}{2 \sqrt{1+k^{\prime 2}}}=\frac{\sqrt{v^{2}+k^{2}}-k}{2 \sqrt{v^{2}+k^{2}}} \in[0,1] .
$$


Since $\omega_{k}^{1} \approx 2 J k$ for low-energy modes, we obtain the first and the second cumulant of work [12]

$$
\begin{aligned}
& \kappa_{1}=N\left(\mu+\frac{2 J}{\pi} \int_{0}^{\pi} \mathrm{d} k k p_{k}\right)=N\left(\mu-\frac{J}{2 \pi} v^{2} \ln v\right), \\
& \kappa_{2}=\frac{8 N J^{2}}{\pi} \int_{0}^{\pi} \mathrm{d} k k^{2} p_{k}\left(1-p_{k}\right)=2 N v^{2} J^{2} .
\end{aligned}
$$

Also, for $n \geq 3, \kappa_{n} \sim v^{2}$ is recovered. Thus, these results verify Eq. (S15).

\section{E: dynamical quantum phase transition}

Dynamical quantum phase transitions are associated with the nonanalytic behavior of physical quantities as functions of time during the evolution of a quantum system [13, 14]. In this dynamical process, the time acts as a controlling parameter and the time domain is partitioned into different regions, in which the physical quantities are characterized by qualitatively different behaviors. Such a phenomenon can be characterized, for instance, by the rate function of the Loschmidt echo of the 1D transverse Ising chain. It is nonanalytic at critical times [13, 14] when considering the thermodynamic limit. The dynamical quantum phase transitions are also relevant in the characterization of the work fluctuations. In fact, as Refs. [13-15] clarified, a connection between the Loschmidt echo and the work distribution (or its characteristic function) can be established at least in two cases: for a single [14, 15] and for a double $[13,14]$ sudden quench protocol.

Although the quench considered in our letter is linear, we also find nonanalytic behaviors of the CFW $\chi(u)$ for large $u$. Since the variable $u$ can be interpreted as the time of the Loschmidt echo in Refs. [14, 15], we still refer to this phenomenon as a dynamical quantum phase transition. For the 1D transverse Ising model, the nonanalytic behavior occurs when the integrand in Eq. (22) is divergent, i.e., when $1+p_{k}\left(e^{2 i u \omega_{k}^{1}}-1\right)=0$. Then, we obtain the critical times $u^{*}$

$$
u^{*}=\frac{\pi}{2 \omega_{k^{*}}^{1}}\left(n+\frac{1}{2}\right), \quad n=0, \pm 1, \cdots,
$$

and the critical mode $k^{*}=\sqrt{v \ln 2 /(2 \pi J)}$, since $p_{k^{*}}=1 / 2$. However, the integral is still welldefined at the critical times by applying analytic continuation. Actually, the cumulant CFW $\ln \chi(u)$ and its derivative are discontinuous at $u^{*}$, i.e., $\lim _{u \rightarrow u^{*-}} \ln \left[\chi(u) / \chi_{a}(u)\right]=-v^{1 / 2} \sqrt{2 / J} \mathrm{Li}_{3 / 2}(2) /(8 \pi)$, $\lim _{u \rightarrow u^{*}} \ln \left[\chi(u) / \chi_{a}(u)\right]=-v^{1 / 2} \sqrt{2 / J} \overline{\operatorname{Li}_{3 / 2}(2)} /(8 \pi), \lim _{u \rightarrow u^{*-}} \partial_{u} \ln \left[\chi(u) / \chi_{a}(u)\right]=-v^{1 / 2} \sqrt{2 J} \lambda_{1} \operatorname{Li}_{1 / 2}(2) /(4 \pi)$ and $\lim _{u \rightarrow u^{*+}} \partial_{u} \ln \left[\chi(u) / \chi_{a}(u)\right]=-v^{1 / 2} \sqrt{2 J} \lambda_{1} \overline{\operatorname{Li}_{1 / 2}(2)} /(4 \pi)$, where $\bar{z}$ denotes the complex conjugate of $z$. It is worth mentioning that these critical times exist only when the protocol goes through the critical point because without crossing the critical point (e. g., $\lambda_{0}<\lambda_{1}<0$ ), we always have $p_{k}<1 / 2$ for all the modes.

[1] C. P. Sun, Journal of Physics A: Mathematical and General 21(7), 1595 (1988).

[2] G. Rigolin, G. Ortiz, and V. H. Ponce, Phys. Rev. A 78(5), 052508 (2008).

[3] C. De Grandi, and A. Polkovnikov, Quantum Quenching, Annealing and Computation. (pp. 75-114) (Springer, Berlin, Heidelberg, 2010).

[4] C. De Grandi, V. Gritsev, and A. Polkovnikov, Phys. Rev. B 81(1), 012303 (2010).

[5] C. De Grandi, V. Gritsev, and A. Polkovnikov, Phys. Rev. B 81(22), 224301 (2010).

[6] P. Talkner, P. S. Burada and P. Hänggi, Phys. Rev. E 78, 011115 (2008).

[7] J. Dziarmaga, Advances in Physics, 59(6), 1063-1189 (2010).

[8] B. Damski and W. H. Zurek, Phys. Rev. A 73, 063405 (2006).

[9] Z. Y. Fei, and H. T. Quan, Phys. Rev. Research, 1(3), 033175 (2019).

[10] B. Damski, Phys. Rev. E 87(5), 052131 (2013).

[11] S. J. Gu, Int. J. Mod. Phys. B 24, 4371 (2010).

[12] Actually for $\kappa_{2}$, Eq. (S18) overestimates the results compared with numerical results because $p_{k}<\left(\sqrt{v^{2}+k^{2}}-\right.$ $k) /\left(2 \sqrt{v^{2}+k^{2}}\right)$ and $\omega_{k}^{1}<2 J k$ for high-energy modes. But, Eq. (S18) does lead to the correct scaling behavior in the cumulant CFW.

[13] M. Heyl, A. Polkovnikov, and S. Kehrein, Phys. Rev. Lett. 110, 135704 (2013).

[14] N. O. Abeling and S. Kehrein, Phys. Rev. B 93, 104302 (2016).

[15] A. Silva, Phys. Rev. Lett. 101, 120603 (2008). 